\title{
Poorer white matter microstructure predicts slower and more variable reaction time performance: evidence for a neural noise hypothesis in a large lifespan cohort
}

\author{
Abbreviated Title: Poor WM predicts slow and variable RT performance
}

Ethan M. McCormick*1,2,3 Cam-CAN $^{4}$, \& Rogier A. Kievit ${ }^{1,4}$

${ }^{1}$ Cognitive Neuroscience Department, Donders Institute for Brain, Cognition and Behavior, Radboud University Medical Center, Nijmegen, The Netherlands; ${ }^{2}$ Methodology and Statistics Department, Institute of Psychology, Leiden University, Leiden, Netherlands; ${ }^{3}$ Department of Psychology and Neuroscience, University of North Carolina, Chapel Hill, United States; ${ }^{4}$ MRC Cognition and Brain Sciences Unit, University of Cambridge, United Kingdom

*corresponding author Ethan M. McCormick Wassenaarseweg 522333 AK, Leiden, The Netherlands e.m.mccormick@fsw.leidenuniv.nl 


\begin{abstract}
Most prior research has focused on characterizing averages in cognition, brain characteristics, or behavior, and attempting to predict differences in these averages among individuals. However, this overwhelming focus on mean levels may leave us with an incomplete picture of what drives individual differences in behavioral phenotypes by ignoring the variability of behavior around an individual's mean. Particularly, enhanced white matter (WM) structural microstructure has been hypothesized to support consistent behavioral performance by decreasing gaussian noise in signal transfer. Conversely, lower indices of white matter microstructure are associated with greater within-subject variance in deploying performance-related resources, especially in clinical populations. We tested a mechanistic account of a 'neural noise' hypothesis in a large adult lifespan cohort (Cam-CAN) with over 2500 adults (ages 18-102; 1508 female; 1173 male; 2681 behavioral sessions; 708 MRI scans) using WM fractional anisotropy (FA) to predict mean levels and variability in reaction time performance on a simple behavioral task using a dynamic structural equation model (DSEM). By modeling robust and reliable individual differences in within-person variability, we found support for a neural noise hypothesis (Kail, 1997), with lower FA predicted individual differences in separable components of behavioral performance estimated using DSEM, including slower mean responses and increased variability. These effects remained when including age, suggesting consistent effects of WM microstructure across the adult lifespan unique from concurrent effects of ageing. Crucially, we show that variability can be reliably separated from mean performance using advanced modeling tools, enabling tests of distinct hypotheses for each component of performance.
\end{abstract}




\section{Significance Statement}

Human cognitive performance is defined not just by the long-run average, but trial-to-trial variability around that average. However, investigations of cognitive abilities and changes during aging have largely ignored this variability component of behavior. We provide evidence that white matter microstructure predicts individual differences in mean performance and variability in a sample spanning the adult lifespan (18-102). Unlike prior studies of cognitive performance and variability, we modeled variability directly and distinct from mean performance using a dynamic structural equation model, which allows us to de-couple variability from mean performance and other complex features of performance (e.g., autoregression). The effects of WM were robust above the effect of age, highlighting the role of WM in promoting fast and consistent performance. 
Poorer white matter microstructure predicts slower and more variable reaction time performance

\section{Introduction}

Prominent theories of cognitive aging posit a central role for reductions in mental

3 processing speed during later life (Salthouse, 1996). These theories focus primarily on decreases

4 in mean performance (i.e. slowing-down) but mostly ignore variability in processing speed, despite

5 a long acknowledgement that variability around the mean provides unique information (Fiske \&

6 Rice, 1955; Nesselroade, 1991; Woodrow, 1932). Prior work shows that average reaction time

7 (RT) displays steep improvement early in life, followed by gradual slowing into aging (Li et al.,

8 2004). Complementary work shows a similar pattern for behavioral variability (Williams et al.,

9 2005). Crucially, despite these similar patterns, evidence strongly suggests that variability provides

10 unique insight into cognitive aging above and beyond mean performance alone (Dykiert et al.,

11 2012; Eizenman et al., 1997; Gamaldo et al., 2012). For instance, trial-to-trial variability might

12 reflect different strategies, momentary lapses (Adam et al., 2015), or endogeneous differences in

13 the signal-to-noise ratio of neural-evoked responses.

14 The neural noise hypothesis (Kail, 1997) posits that the signal-to-noise ratio of the central

15 nervous system degrades during aging, leading to deficits in processing speed.

16 Electrophysiological recordings have shown that increased neural noise (i.e., de-synchronized

17 neural oscillations) led to slower behavioral performance in older adults (Dave et al., 2018; Voytek

18 et al., 2015) due to disrupted long-range communication and synchronization between neural

19 regions (Voytek \& Knight, 2015). Reduced inter-region communication suggests a causal role for

20 demyelination of neuronal axons carrying signals between cortical regions (Peters, 2009). While

21 the mechanisms of progressive demyelination leading to slowing of RT is debated (Bartzokis et

22 al., 2010; Peters, 2009), this relationship is robust in normative samples (Bennett \& Madden, 2014;

23 Fjell et al., 2011; Tamnes et al., 2012; Turken et al., 2008). Additionally, in clinical samples with 
24 specific focal damage to white matter (e.g., multiple sclerosis), increased white matter damage is

25 associated with increased behavioral variability (Britton et al., 1991; Bunce et al., 2007). While

26 prior findings have focused primarily on mean processing speed (but see Li \& Lindenberger,

27 1999), this logic extends naturally to variability (Halliday et al., 2019; MacDonald et al., 2006;

28 Sorg et al., 2021). Impaired white matter is more prone to random leakage from axonal signals that

29 lead to inconsistencies in behavioral performance. As such, we hypothesize that reduced white

30 matter measures should predict slower overall performance and increased trial-to-trial variability.

31 A key limitation in prior work on variability is the widespread use of measures such as the

32 standard deviation of response times (iSD) or coefficient of variation (iCV; equal to the iSD

33 divided by the mean) across trials (e.g., Haynes et al., 2017). Unfortunately, these simple estimates

34 of variability fail to account for complex features of behavioral performance (e.g., trends or

35 autoregression), leading to systematic over- or under-estimates of variability (Wang \& Grimm,

36 2012). To address these challenges, we employed dynamic structural equation modeling (DSEM;

37 Asparouhov et al., 2018), which combines strengths of timeseries analysis with hierarchical

38 random effects in a structural equation framework. This approach allows us to test trial-to-trial

39 predictors of RT data at the within-person level, and explain person-to-person differences in mean

40 RT and variability simultaneously (McNeish \& Hamaker, 2020). Using this innovative framework

41 for capturing variability de-confounds sources of variance that might otherwise bias individual

42 differences performance features, and allows us to test different causal mechanisms associated

43 with individual differences in mean and variability in RT.

44 We tested this neural noise hypothesis in a large cohort of adults (age 18-102; Shafto et

45 al., 2014) who completed a set of reaction-time tasks $(\mathrm{N}=2681)$ and underwent a diffusion-

46 weighted scan $(\mathrm{N}=708)$. We modeled individual differences in four aspects of behavioral 
47 performance: mean RT, variability, trends across the task, and the autoregressive effect between

48 adjacent trials. We incorporated differences in white matter and age to examine how they predicted

49 each component of processing speed performance. We hypothesized that reduced WM

50 microstructure would predict increased mean RT and heightened variability (i.e., slower, less

51 consistent performance), and that these effects would be robust to including age in the model. In

52 doing so, we pursued dual goals: 1) to conduct a theoretical test of the neural noise hypothesis, and

53 2) to use that test to outline a generative DSEM framework for testing hypotheses related to

54 variability for future work in this area.

\section{Methods}

For consistency and precision, portions of the text in this section are drawn from prior work describing the Cambridge Centre for Ageing and Neuroscience (Cam-CAN) dataset (Shafto et al.,

59 2014; Taylor et al., 2017).

60 Participants

61 Data were provided by healthy adult participants in two phases of data collection. Stage 1 62 consisted of at-home interviews of individuals living in the Cambridge City, UK region. During

63 Stage II, a subset of these participants was invited to complete a neuroimaging session, where

64 structural and functional scans were obtained. Participants at Stage II were recruited in deciles

65 between 18-87 years of age, with a goal of equal sample sizes ( 100) at each decile. In the current 66 analyses, we utilized behavioral data (SRT) from 2681 healthy adults (1508 female; 1173 male)

67 from Stage I and additional behavioral data (VSTM) and measures of structural WM from 708

68 adults (359 female; 349 male) from Stage II. Note that a subset of these data have been analysed

69 before in Kievit et al (2016) using more simplistic methods. Exclusion criteria included MR safety 
70 contraindications (e.g., pacemakers), learning disability (living at home), cognitive impairment

71 (Mini-Mental State Examination (MMSE; Folstein et al., 1975) score of 24 or less) and reduced

72 response from individuals with limited longstanding illness or disability (for full information on

73 exclusion criteria, see Table 1 in Shafto et al., 2014). Prior to the interview, individuals give written

74 informed consent for the study and record linkage. Individuals who lack the capacity to give

75 consent were not included. Written informed consent was also given by participants at each session

76 for Stages 2 and 3 (Shafto et al., 2014).

\section{Experimental Design}

\section{Behavioral Tasks}

The description of the behavioral tasks is copied, in part, from Shafto and colleagues

80 (2014). For additional information regarding the tasks and requests for data access, see the Cam-

81 CAN dataset inventory (https://camcan-archive.mrc-cbu.cam.ac.uk/dataaccess/).

82 Simple Response Time Task. A "simple" response time task (SRT) was used to assess basic

83 aspects of speeded responses. In the SRT, participants viewed an image of a hand with blank circles

84 above each finger, while resting their right hand on a response box with four buttons, one for each

85 finger. When a given circle turned black on the image, the participants were instructed to press

86 with the relevant finger as quickly as possible. On pressing the button (or after a maximum of 3

87 seconds) the circle became blank again and the variable inter-trial interval (ITI) began. The ITI

88 was varied pseudo-randomly with a positively skewed distribution (mean $=3.7 \mathrm{~s}$, median $=3.9 \mathrm{~s}$,

89 range $=1.8-6.8 \mathrm{~s}$ ). The SRT consisted of 50 trials, and the principal outcome measure was

90 reaction time from stimulus onset to button press. For a visualization of the task, see Figure 2a in

91 Kievit and colleagues (2016).

92 Visual Short Term Memory Task. This task assesses the processes underpinning visual 
93 short-term memory (VSTM). In the VSTM, participants viewed a series of circular discs presented

94 briefly on a computer screen. After a brief delay, participants reported the color of a cued disc, by

95 selecting from a color wheel that displays a rainbow of hues. On each trial, participants saw a

96 display for 250 milliseconds which contains a central fixation and one to four colored discs, with

97 the colours chosen at random. The locations of the discs on the screen were randomly selected

98 from eight points equidistant from a central fixation. Following the brief encoding display, there

99 was a 900-millisecond blank screen, and then one of the disc locations was highlighted with a

100 border and the response colour wheel appeared. On half of trials, any un-cued discs also

101 reappeared, to provide the context within which the disc was encoded. Participants indicated their

102 confidence in the selected colour by the length of time they held down their finger: as they held

103 their finger down for longer, white confidence intervals spread out around the selected point

104 indicating more uncertainty about their selection. The response interval did not have a time limit:

105 after participants confirmed their response, there was an 830-millisecond fixation period before

106 the next trial began. After a brief practice period, participants completed two blocks of 112 trials,

107 with set-size and probe context being counterbalanced and randomly intermixed within each.

108 Participants also completed a perceptual control block of 56 trials, where single discs were

109 presented at fixation along with the colour wheel, until the participant matched their hue by

110 selecting the appropriate point on the surrounding wheel. There were two possible responses to

111 model with the VSTM data, a first response and a final response. In general, we discuss results

112 related to the final response, but full results from both models are available (https://osf.io/nkjdt/).

113 We note major differences if relevant.

\section{White Matter Microstructure}


White matter microstructure was assessed using the fractional anisotropy (FA) of WM

116 tracts derived from diffusion weighted imaging - see prior work for full details on the relevant

117 scan parameters (Taylor et al., 2017) and processing pipeline (Kievit et al., 2016). We utilized FA

118 given its widespread use in investigations of aging and white matter, however, it should be noted

119 that FA is a complex measure and its exact relation to white matter health is not fully understood

120 (Jones et al., 2013). Furthermore, its relatively simple model structure may not capture especially

121 complex features of white matter organization (e.g., crossing fiber bundles). Consistent with prior

122 work, mean FA was computed for the ten tracts defined by the Johns Hopkins University white 123 matter tractography atlas (Hua et al., 2008; see Kievit et al., 2016 for tract visualization): the

124 anterior thalamic radiations (ATR), corticospinal tract (CST), dorsal cingulate gyrus (CING), 125 ventral cingulate gyrus (CINGHipp), forceps major (FMaj), forceps minor (FMin), inferior fronto126 occipital fasciculus (IFOF), inferior longitudinal fasciculus (ILF), superior longitudinal fasciculus 127 (SLF), and uncinate fasciculus (UNC). As a sensitivity analysis, FA values more than 4 standard 128 deviations from the mean in each tract ( $0.27 \%$ of values) were excluded for the core set of analyses. 129 Model results were not substantively impacted and so all values were retained in the presented 130 results.

131 We faced a challenge when using the different WM tracts as predictors in the DSEM 132 (detailed below), due to the high correlations across measures (54\% of the total variance explained 133 by the first principal component). We considered several options, but each came with limitations:

134 1) PCA-derived scores did not allow for missing data, 2) WM tracts do not theoretically conform 135 to a reflective latent variable structure, and 3) including all tracts as simultaneous predictors 136 resulted in unstable results due to the high multi-collinearity (Lavery et al., 2019). Furthermore, 137 prior work (de Mooij et al., 2018) showed that while white matter tracts are correlated, they do not 
Poorer white matter microstructure predicts slower and more variable reaction time performance

138 show a unidimensional or simple factor structure (i.e., predominant loading onto a single factor, 139 negative factor loadings, etc.). As such, we fit models for each tract separately and looked across 140 models for consistent findings. We thereby present a single tract (the ATR) as a canonical example,

141 while noting any deviation from the canonical pattern of effects if needed. We do not attempt to

142 draw specific theoretical inferences for individual tracts, but rather take them as repeated instances

143 of a WM-general effect. Consistent effects across tracts would indicate that the effects are 144 generally related to WM fractional anisotropy, while inconsistent effects might suggest functional

145 specificity - which we do not expect.

\section{$146 \quad \underline{\text { Statistical Analysis }}$}

\section{Dynamic Structural Equation Model}

All models were fit using the DSEM module in Mplus Version 8 for intensive longitudinal

149 data (Asparouhov et al., 2018). DSEM is a flexible modeling framework which combines strengths

150 of traditional time-series analyses, multilevel models, and structural equation models (for a primer

151 on DSEM, see (McNeish \& Hamaker, 2020). DSEM also allows for the simultaneous estimation

152 of the random effects of behavioral performance and their association with covariates of interest,

153 rather than a 2-step approach that does not propagate the uncertainty in estimates across levels of

154 analysis (Wang \& Grimm, 2012). At the within-person trial level, we modeled the natural log of

155 reaction time $(\operatorname{logRT})$ for both SRT and VSTM behavior on a given trial as a function of reaction

156 time at the prior trial $(\varphi)$ and the trial number $\left(\beta_{t}\right)$. Phi $(\varphi)$ represents the autoregressive effect, or

157 inertia of performance between adjacent trials. Individuals with high autoregressive effects would 158 show slower cycles of faster and slower performance around their mean, while smaller 159 autoregressive effects would reflect larger differences in performance between adjacent trials 160 (compare the grey and pink exemplar timeseries in Figure 1B). The trend $\left(\beta_{t}\right)$ parameter reflects 
161 overall gains or losses in performance across the task session (here the trend across trials but this 162 could reflect other metrics of time; McCormick, 2021; compare the grey and blue exemplar

163 timeseries in Figure 1B). At the person level, we modeled random effects for $\varphi$ and the trend $\left(\beta_{t}\right)$,

164 as well as for the mean $(\mu)$ and variance $(\psi)$ of RT across the task. By default the between-person

$165 \psi$ parameter was estimated with a log-linear model to aid in estimation (McNeish \& Hamaker, 166 2020). For each task, we compared a fixed-effects model to one where each of these parameters 167 was allowed to vary freely. To assess the robustness of the results and compare models, we took 168 several steps to evaluate the results. Model convergence was achieved when the potential scale 169 reduction (PSR) metric, which estimates the potential reduction in the width of the posterior 170 parameter distribution with infinite subsequent draws, was less than 1.10 (Gelman \& Rubin, 1992), 171 reflecting less than a $10 \%$ potential narrowing compared the current distribution. Trace plots 172 associated with the estimated parameter were examined visually to ensure that chains converged 173 to a random scatter around a fixed mean (i.e., flat traces). To ensure model robustness, we 174 compared a solution with 10,000 iterations to one with 20,000 iterations using the same random 175 seed. Parameter changes between models were assess with a measure of relative bias (i.e., the 176 difference between the two parameter solutions divided by the original parameter). We then 177 estimated the same model with 20,000 iterations twice more, with different random seeds to assess 178 the impact of starting values on the final solution. If bias was low $(<5 \%)$ across solutions, we 179 retained the model; otherwise, we doubled the iterations for all solutions and assessed relative bias 180 on the new models. All models presented here converged to acceptable values (for all model 181 diagnostics and comparisons, see the analysis code; https://osf.io/nkjdt/). To correct for the 182 multiple models, we applied false discovery rate correction to the significance tests. Because the 
Poorer white matter microstructure predicts slower and more variable reaction time performance

183 standard Mplus output only gives floating point precision to the third decimal place, we calculated

184 the adjusted $p$-values from the estimate and posterior standard error.

185 We then built a series of conditional models predicting the random effects by including the

186 white matter tract FA (with the ATR as the canonical example) and age separately before including

187 both in a final conditional model. The unconditional model can be expressed in the multilevel

188 equation below, for person $i$ at trial $t$ (colors included to help keep track of the different components

189 of behavior).

190

Level 1:

$$
\begin{aligned}
& \log \left(R T_{t, i}\right)=\mu_{i}+\varphi_{i} R T_{t-1, i}+\beta_{t i} \operatorname{Trial}_{t, 1}+\varepsilon_{t, i} \\
& \varepsilon_{t, i} \sim N\left(0, \psi_{i}\right)
\end{aligned}
$$

Level 2:

$$
\begin{aligned}
& \mu_{i}=\gamma_{00}+u_{0 i} \\
& \varphi_{i}=\gamma_{10}+u_{1 i} \\
& \beta_{t i}=\gamma_{20}+u_{2 i} \\
& \psi_{i}=\exp \left(\omega_{0}+u_{3 i}\right)
\end{aligned}
$$

$$
\mathbf{u}_{i} \sim M V N\left(\left[\begin{array}{l}
0 \\
0 \\
0 \\
0
\end{array}\right],\left[\begin{array}{llll}
\tau_{\mu} & & \\
\tau_{21} & \tau_{\varphi} & \\
\tau_{31} & \tau_{32} & \tau_{\beta_{t}} & \\
\tau_{41} & \tau_{42} & \tau_{43} & \tau_{\psi}
\end{array}\right]\right)
$$

191

192 With covariates entering the model at Level 2 for the conditional model:

193

Level 2:

$$
\begin{aligned}
& \mu_{i}=\gamma_{00}+\gamma_{01} W M_{i}+\gamma_{02} A_{g e_{i}}+u_{0 i} \\
& \varphi_{i}=\gamma_{10}+\gamma_{11} W M_{i}+\gamma_{12} A g e_{i}+u_{1 i} \\
& \beta_{t i}=\gamma_{20}+\gamma_{21} W M_{i}+\gamma_{22} A g e_{i}+u_{2 i} \\
& \psi_{i}=\exp \left(\omega_{0}+\omega_{1} W M_{i}+\omega_{2} A_{g} e_{i}+u_{3 i}\right)
\end{aligned}
$$




$$
\mathbf{u}_{i} \sim M V N\left(\left[\begin{array}{l}
0 \\
0 \\
0 \\
0
\end{array}\right],\left[\begin{array}{llll}
\tau_{\mu} & & \\
\tau_{21} & \tau_{\varphi} & \\
\tau_{31} & \tau_{32} & \tau_{\beta_{t}} & \\
\tau_{41} & \tau_{42} & \tau_{43} & \tau_{\psi}
\end{array}\right]\right)
$$

195 Note that in the conditional model, the elements in $\boldsymbol{u}_{\mathrm{i}}$ are residual (co)variances. We can also

A
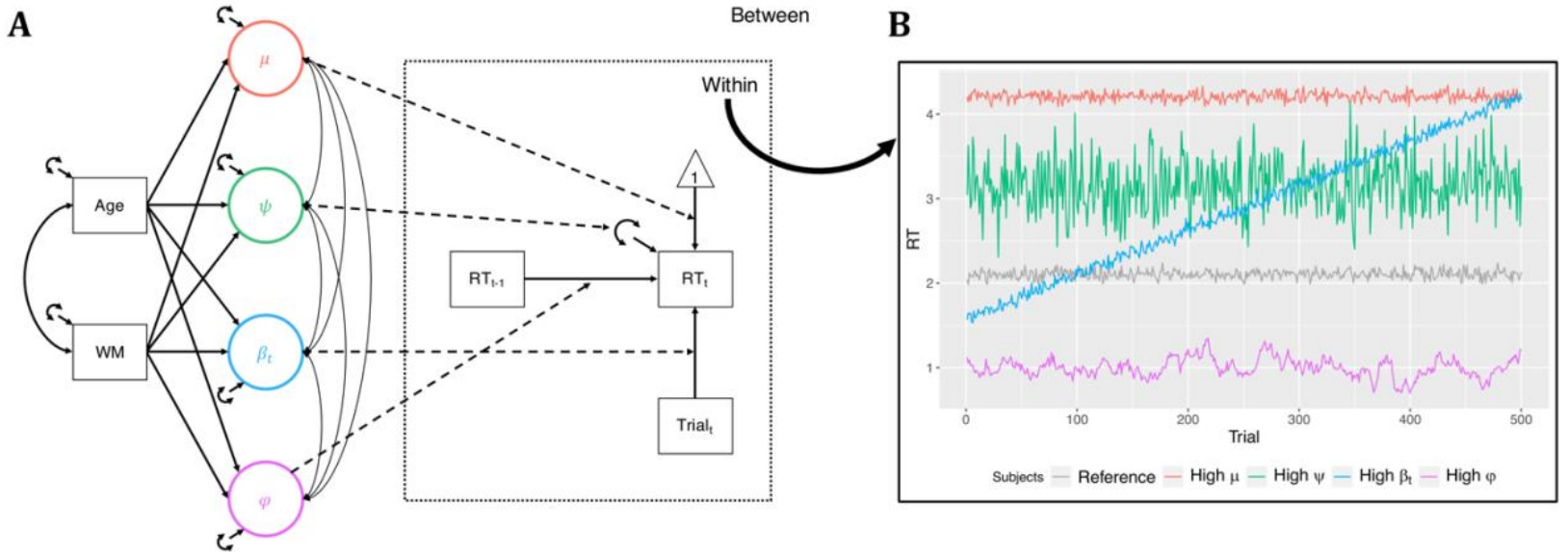

Figure 1. A) Model Schematic. We estimated 4 random effects $(\mu, \psi$, and $\varphi)$ at the between-person level from the within-person model (dashed lines) of reaction time $\left(R T_{t}\right)$ regressed on its prior value $\left(R T_{t-1}\right)$ and the current trial number (Trial $t_{t}$. Observed variables are depicted in boxes and latent variables in circles as is conventional. The random effects were regressed (solid single-headed arrows) on the covariates, Age and WM fractional anisotropy (tracts were modeled individually so the identity of this covariate varied over models). Finally, the covariance between Age and FA (solid double-headed arrow, left) and residual covariances between the random effects (solid doubleheaded arrows, right) were estimated. B) Exemplar Timeseries. We simulated exemplar timeseries to highlight the impact of higher values on each of the 4 random effects. The exemplars differed from the reference timeseries (grey) in mean (red; High $\mu$ ), variance (green; High $\psi$ ), trend (blue; High $\beta_{t}$ ), and autoregressive (purple; High $\varphi$ ) parameters.

212 The inclusion of age served as an important covariate to ensure that our results were not driven

213 solely by systematic differences across age in mean RT $(r=0.242)$ and co-occuring age and WM

214 FA $(r=-0.470)$ across the lifespan. Because only a subset of participants who contributed data at 
Poorer white matter microstructure predicts slower and more variable reaction time performance

215 Stage I (SRT) were invited to Stage II (VSTM and WM measures), we used a joint likelihood

216 approach instead of the default conditional likelihood to allow missingness on exogenous variables

217 without listwise deletion by estimating the mean and variance of each predictor (i.e., age and WM

218 tract FA). The raw data are available upon signing a data sharing request form (see

219 http://www.mrc-cbu.cam.ac.uk.libproxy.lib.unc.edu/datasets/camcan/ for more detail). Analysis

220 code and full model results are available on OSF (https://doi.org/10.17605/OSF.IO/BN6YU).

221

222

223

224

225

226

227

228

229

230

231

232

233

234

235

236

237

238

\section{Results}

\section{Unconditional Model}

We began by fitting two unconditional models to the SRT data, one with only fixed (i.e., zero variance) effects and the other with four random effects. The deviance information criteria $\left(\mathrm{DIC}_{\text {fixed }}=1793, \mathrm{DIC}_{\text {random }}=-44107, \Delta \mathrm{DIC}=-45900\right)$ overwhelmingly favored the inclusion of the four random effects (Asparouhov et al., 2018; McNeish \& Hamaker, 2020). Fixed effects-only models in the VSTM data also showed very poor fit bolstering the rationale for adopting a random effects approach to capture individual variability in the different components of the RT data. In a follow-up analyses, we compared the full random effect model to a set of models where we constrained each random effect to zero in turn. Model comparisons provided strong evidence in favor of including all random effects ( $\triangle$ DICs ranging from -193 to -20222). In other words, the model fit results suggested that the complexity of the behavioral data could not be appropriately captured by a single effect that held for all members of the sample and rather that allowing for individual differences in all four components of performance was necessary. For the SRT data, a model with 4 random effects showed that there is significant person-to-person variability in all 4 of the parameters modeled (Table 1). Examining the average within-person standardized effects (Schuurman et al., 2016), there was small positive inertia in participants' response time across 
239 adjacent trials $\left(\mathrm{RT}_{\mathrm{t}-1} ; \beta=0.036, S D_{\text {posterior }}=0.003,95 \%\right.$ C.I. $\left.=[0.030,0.042]\right)$ and participants got

240 faster on average across the task (Trialt; $\beta=-0.062, S D_{\text {posterior }}=0.003,95 \%$ C.I. $\left.=[-0.067,-0.056]\right)$.

241 Results for the VSTM data confirmed this same general pattern.

242 When examining the pattern of correlations there was some heterogeneity between the

243 tasks. In the SRT data, the mean $(\mu)$, variability $(\psi)$, and autoregressive $(\varphi)$ random effects were

244 all positively correlated with one another, and all negatively correlated with the trend effect $\left(\beta_{t}\right.$;

245 see Figure 2). Of particular note was the correlation between mean RT and response variability

246 which was moderately positive $(r=0.397,95 \%$ C.I. $=[.359, .434])$, suggesting that we could

247 achieve separation of individuals' mean and variability in performance (compared with $r=0.666$

248 between mean RT and a simple iSD measure). Interestingly, mean RT in the VSTM data showed

249 a different pattern of relations (Final Response: $r=-0.398,95 \%$ C.I. $=[-0.465,-0.328]$ ), being

250 negatively correlated with the other random effects. This perhaps indicates more of a ceiling effect,

251 rather than the floor effect seen in simple speeded response tasks. 


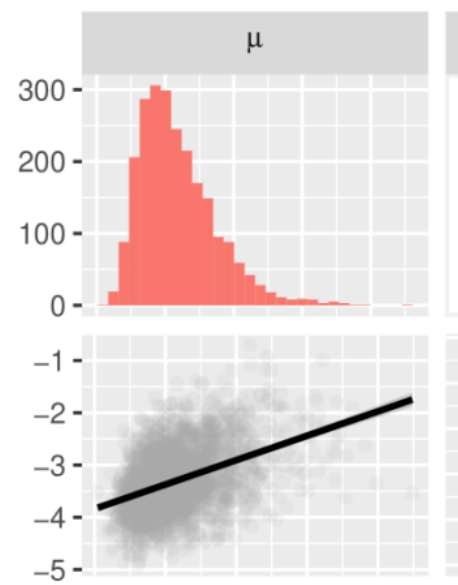

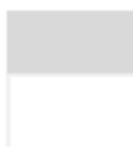

$\psi$

\section{Corr:}

$0.397^{\star * \star}$
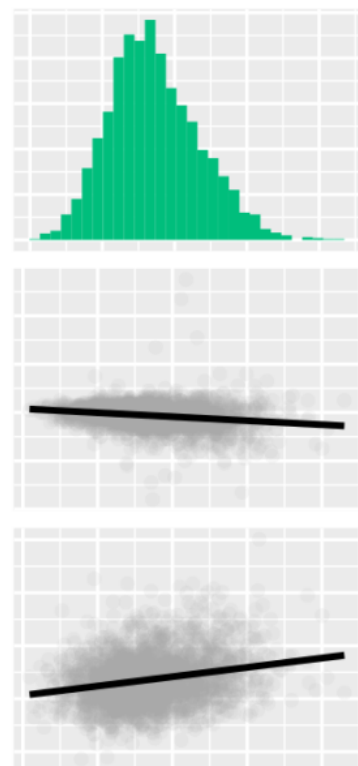

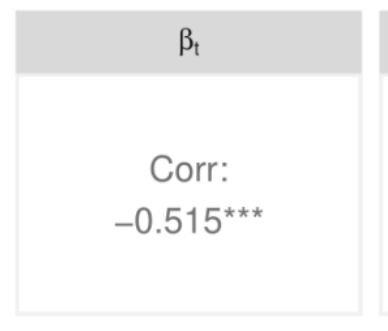

Corr: $-0.181^{\star \star *}$
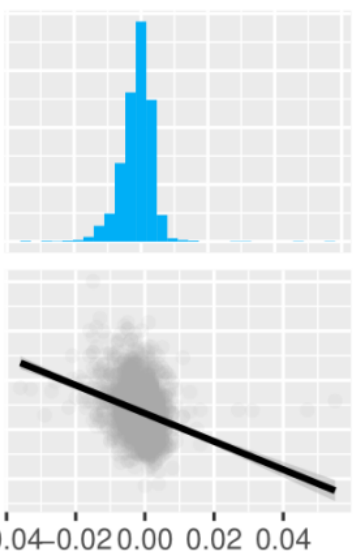

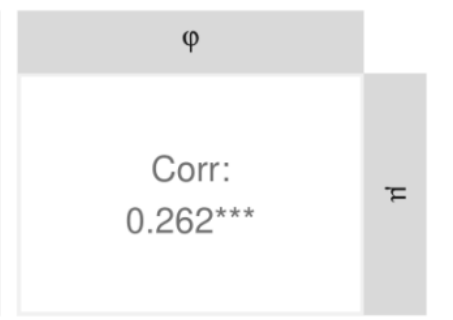

Figure 2. Distributions of Random Effects from the Simple RT task. Each of the four random effects showed significant between-person variability. Mean performance $(\mu)$, variability $(\psi)$, and inertia $(\varphi$; i.e., the autoregressive effect $)$ were all positively correlated, and negatively correlated with the trend of performance across the task $\left(\beta_{t}\right)$.

Model Based and Observed Measures of Variability

To further clarify the relationship between the DSEM-derived components of behavioral performance and traditional measures calculated directly from the data (iSD and iCV). We calculated these observed measures on the trial level RT values after taking the natural log and entered the factor score estimates of the random effects as predictors in a multiple regression analysis (Figure 3). The iSD measure was significantly predicted by all four random effects, while

263 the iCV measure was significantly predicted by the mean, variability, and trend but not inertia factor scores. Interestingly, while variability strongly related to iSD $(\beta=0.885, S E=0.007, p<$ 
$.001)$, it weakly and negatively related to $\mathrm{iCV}(\beta=-0.167, S E=0.021, p<.001)$. Indeed, iCV was explained in iCV was relatively low $\left(R^{2}=0.111\right)$, especially compared with the high variance
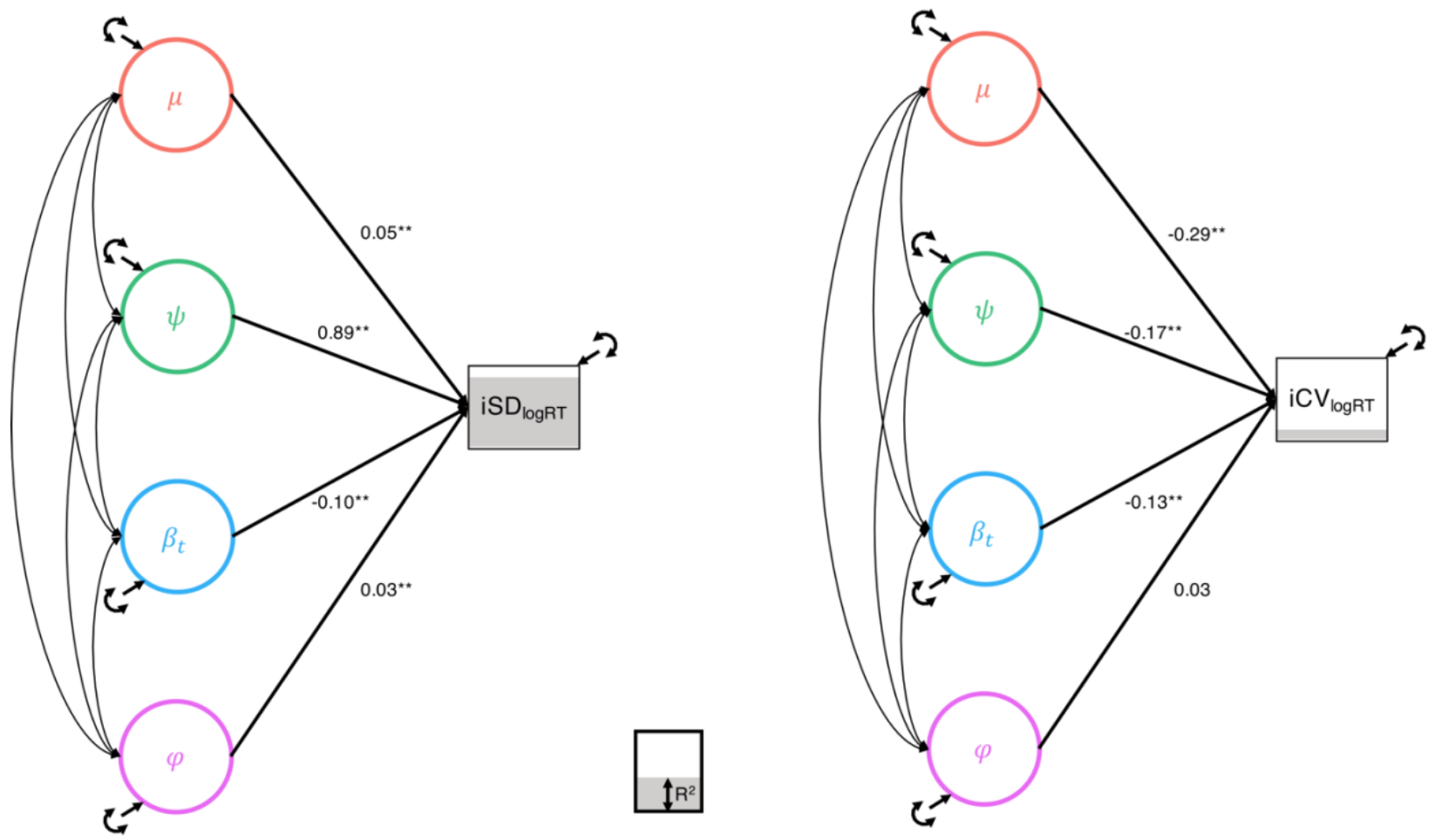

Figure 3. Explaining Observed Metrics of Interindividual Variability in SRT. While iSD was well described by the linear combination of the four random effects, the $i C V$ showed surprising negative relationships with the metrics of mean and variability in performance derived from the dynamic structural equation model. However, both metrics showed significant contamination by more than one random effect, especially mean performance $(\mu)$ and the trend $\left(\beta_{t}\right)$.

\section{Conditional Model without Age}

We next included the 10 white matter tracts as predictors in separate models at the between- 
tracts (all models for the other tracts are available at https://osf.io/nkjdt/). As hypothesized, reduced white matter FA predicted both slower response times $\left(\beta=-0.322, S D_{\text {posterior }}=0.042,95 \%\right.$ C.I. $=[-0.401,-0.237])$ and increased response variability $\left(\beta=-0.344, S D_{\text {posterior }}=0.042,95 \%\right.$ C.I.

$285=[-0.423,-0.260])$. Furthermore, lower white matter FA predicted a higher autoregressive effect $286\left(\beta=-0.123, S D_{\text {posterior }}=0.053,95 \%\right.$ C.I. $\left.=[-0.229,-0.021]\right)$ and a less positive trend $(\beta=0.119$, $287 S D_{\text {posterior }}=0.034,95 \%$ C.I. $\left.=[0.053,0.186]\right)$. The addition of the ATR covariate reduced the 288 residual correlation between mean RT and response variability $\left(r^{\prime}=0.263\right.$, 95\% C.I. $=[.268$, $289.371]$ ). The other tracts had similar effects (although the effects for the ventral cingulate gyrus 290 [CINGHipp] were not significant) in the SRT data. In the VSTM data, reduced ATR FA predicted 291 only slower mean RT $\left(\beta=-0.919, S D_{\text {posterior }}=0.344,95 \%\right.$ C.I. $\left.=[-1.581,-0.239]\right)$ but not response 292 variability $\left(\beta=-0.653, S D_{\text {posterior }}=0.787,95 \%\right.$ C.I. $=[-2.198,0.878]$.
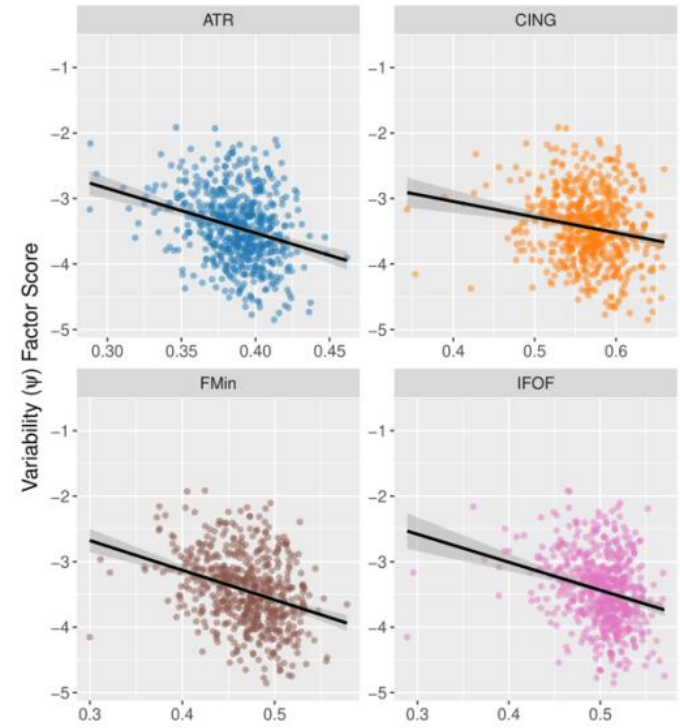

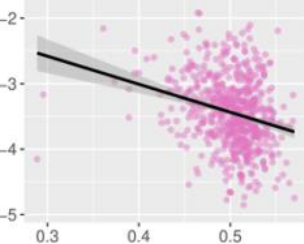

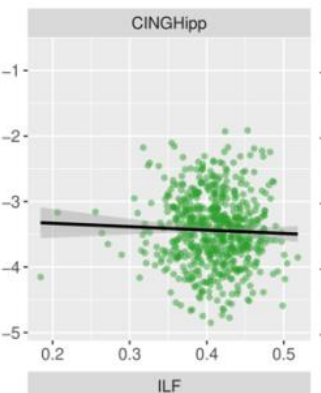
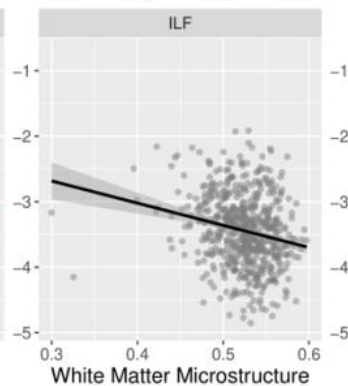

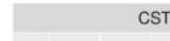

ST
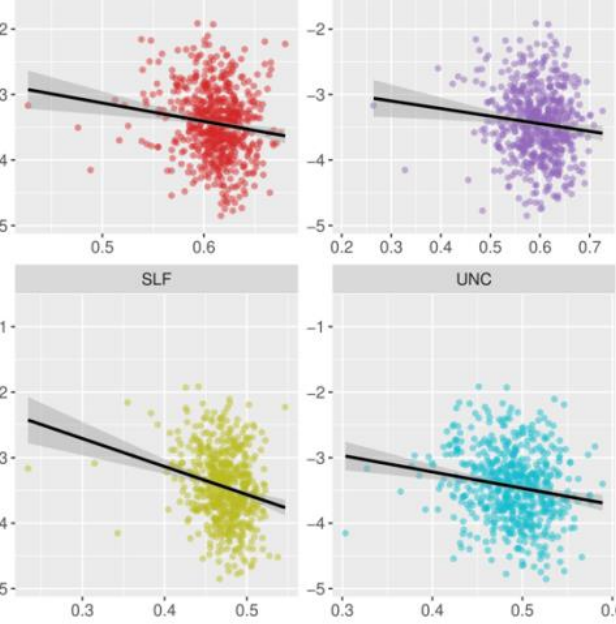

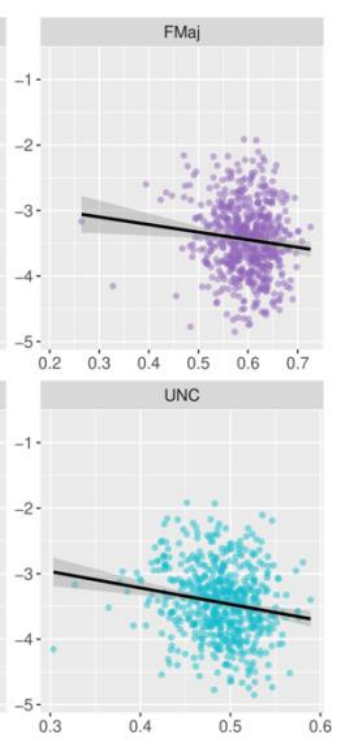

Figure 4. White Matter Microstructure and Variability. Across nine of the ten canonical tracts, reduced white matter fractional anisotropy predicted more variability in response times (in the CINGHipp tract, there was no significant relationship). 
Finally, we included age as an additional covariate predicting the random effects to account

300 for the likely confounding of systematic WM FA variation decreases across the adult lifespan

301 (Figure 1). Results suggested that older individuals showed slower average RT $(\beta=0.351, S D$ -

302 posterior $=0.032,95 \%$ C.I. $=0.287,0.413])$ and greater response variability $\left(\beta=0.272, S D_{\text {posterior }}=\right.$

$3030.034,95 \%$ C.I. $=0.204,0.337]$; Table 1; Figure 5). Although somewhat attenuated compared to

304 the conditional model without age, lower white matter FA continued to uniquely predict slower 305 mean performance $\left(\beta=-0.149, S D_{\text {posterior }}=0.049,95 \%\right.$ C.I. $\left.=[-0.244,-0.051]\right)$ and increased 306 behavioral variability $\left(\beta=-0.197, S D_{\text {posterior }}=0.051,95 \%\right.$ C.I. $\left.=[-0.296,-0.095]\right)$. The inclusion 307 of age and WM FA together explained 17-20\% of the between-person variance in mean RT $\left(R^{2}=\right.$ $3080.201, S D_{\text {posterior }}=0.018,95 \%$ C.I. $\left.=[0.167,0.238]\right)$ and RT variability $\left(R^{2}=0.169, S D_{\text {posterior }}=\right.$ $3090.020,95 \%$ C.I. $=[0.133,0.212])$, but only $1 \%$ of the variance in the trend across trials $\left(R^{2}=0.012\right.$, $310 S D_{\text {posterior }}=0.013,95 \%$ C.I. $\left.=[0.003,0.041]\right)$ and the variance in the autoregressive effect $\left(R^{2}=\right.$ $3110.010, S D_{\text {posterior }}=0.010,95 \%$ C.I. $=[0.001,0.038]$ ). At the within-person level, the lag-1 (i.e., 312 autoregression) and trial (i.e., trend) effects accounted for $16 \%$ of the variance in individual trial 313 RT $\left(R^{2}=0.163, S D_{\text {posterior }}=0.003,95 \%\right.$ C.I. $\left.=[0.158,0.168]\right)$. 


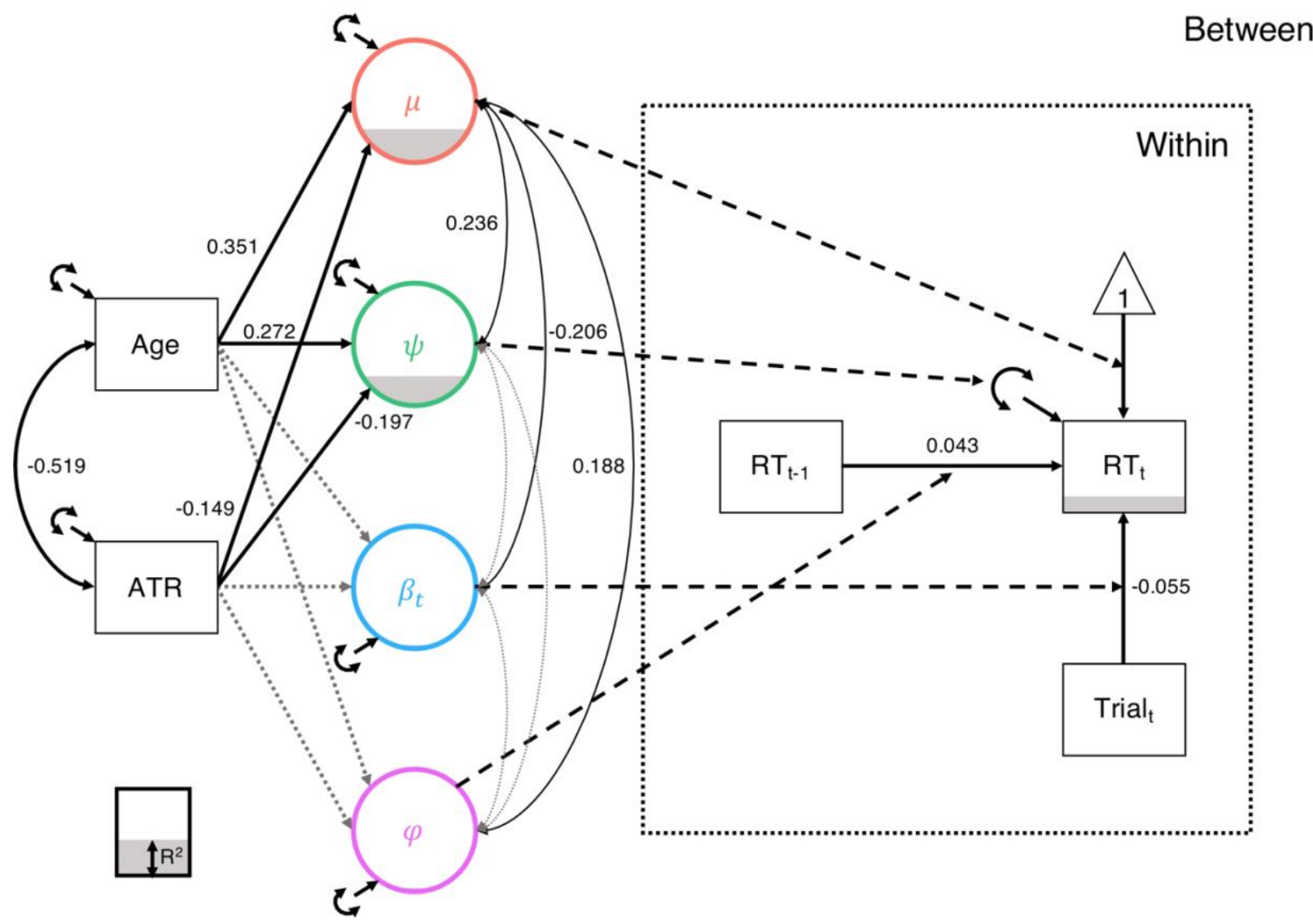

Figure 5. Conditional Model of Simple RT task with Age and White Matter Microstructure. Age and white matter FA had opposite predictive effects, with older individuals showing slower and more variable responding while those with greater white matter FA showed the opposite pattern (i.e., faster, more consistent responding). Paths in red represent positive unique effects or correlations, while those in blue represent negative relationships. The dashed blue line represents a non-significant effect of ATR FA on the autoregressive random effect $(\varphi)$. Explained variance (i.e., $R^{2}$ ) is represented by the proportion of the outcome variable shaded in grey. Dashed black lines link random effects at the between-person level with their corresponding effect at the within-person level.

Table 1. Model Results for Unconditional and Full Conditional Model.

\section{Unconditional Model}

Full Conditional Model

\begin{tabular}{|c|c|c|c|c|c|c|c|c|}
\hline & Est & S.D.post & Lower & Upper & Est & S.D.post & Lower & Upper \\
\hline \multicolumn{9}{|c|}{ Within-Person Standardized Effects } \\
\hline$\gamma_{10}$ & 0.036 & 0.003 & 0.030 & 0.079 & 0.043 & 0.003 & 0.036 & 0.049 \\
\hline$\gamma_{20}$ & -0.053 & 0.003 & -0.059 & -0.047 & -0.055 & 0.005 & -0.063 & -0.044 \\
\hline
\end{tabular}

Between-Person Unstandardized Effects
$\gamma_{00}$
$-0.908$
0.006
$-0.920$
$-0.896$
$-0.495$
$0.253 \quad-0.996$
$-0.008$ 
McCormick, Cam-CAN, \& Kievit

$\begin{array}{lllllllll}\gamma_{10} & 0.036 & 0.004 & 0.029 & 0.043 & 0.196 & 0.128 & -0.059 & 0.446 \\ \gamma_{20} & -0.001 & 0.000 & -0.002 & -0.001 & -0.007 & 0.016 & -0.041 & 0.022 \\ \omega_{0} & -3.292 & 0.014 & -3.309 & -3.254 & -1.689 & 0.594 & -2.880 & -0.536 \\ \tau_{\mu} & 0.083 & 0.003 & 0.078 & 0.089 & 0.066 & 0.002 & 0.062 & 0.071 \\ \tau_{\varphi} & 0.008 & 0.001 & 0.006 & 0.010 & 0.013 & 0.001 & 0.011 & 0.015 \\ \tau_{\beta} & 1.9 \mathrm{e}-5 & 5.1 \mathrm{e}-7 & 1.8 \mathrm{e}-5 & 2.0 \mathrm{e}-5 & 1.7 \mathrm{e}-5 & 6.4 \mathrm{e}-7 & 1.5 \mathrm{e}-5 & 1.8 \mathrm{e}-5 \\ \tau_{\psi} & 0.424 & 0.014 & 0.398 & 0.452 & 0.356 & 0.013 & 0.330 & 0.382\end{array}$

Between-Person Standardized Covariate Effects

ATR FA

$\begin{array}{lllll}\gamma_{01} & -0.149 & 0.049 & -0.244 & -0.051 \\ \gamma_{11} & -0.085 & 0.063 & -0.209 & 0.040 \\ \gamma_{21} & 0.042 & 0.074 & -0.096 & 0.194 \\ \omega_{1} & -0.197 & 0.051 & -0.296 & -0.095 \\ \gamma_{02} & & & & \\ \gamma_{12} & & & & \\ \gamma_{22} & 0.351 & 0.032 & 0.287 & 0.413 \\ \omega_{2} & 0.012 & 0.047 & -0.082 & 0.105 \\ & -0.072 & 0.045 & -0.157 & 0.020 \\ & 0.272 & 0.034 & 0.204 & 0.337\end{array}$

Variance Explained $\left(R^{2}\right)$

\begin{tabular}{|c|c|c|c|c|c|c|c|c|}
\hline $\log \left(R T_{t, i}\right)$ & 0.096 & 0.001 & 0.094 & 0.099 & 0.163 & 0.003 & 0.158 & 0.168 \\
\hline$\mu_{i}$ & & & & & 0.201 & 0.018 & 0.167 & 0.238 \\
\hline$\varphi_{i}$ & & & & & 0.010 & 0.010 & 0.001 & 0.038 \\
\hline$\beta_{t i}$ & & & & & 0.013 & 0.020 & 0.003 & 0.041 \\
\hline$\psi_{i}$ & & & & & 0.169 & 0.020 & 0.133 & 0.212 \\
\hline
\end{tabular}

324 Note: Est. is the sample-recovered parameter. Std. Err. is the standard error of the estimate.

325 S.D.post is the posterior standard deviation. Lower and Upper are the boundaries of the credible 326 intervals. 
Poorer white matter microstructure predicts slower and more variable reaction time performance

\section{Discussion}

In the modeling of human behavior, analysis of mean performance has preoccupied the

329 majority of research focus. Here, we offer compelling evidence that variability in performance

330 holds unique additional value for a broader understanding of human behavior. Crucially, to

331 accurately isolate the mechanisms and consequences of this parameter as distinct from the mean

332 requires appropriate quantitative tools. Utilizing a dynamic structural equation model (DSEM;

333 Asparouhov et al., 2018) approach, we fit a model that separates out four distinct components of

334 human reaction time behavior: mean performance, variability in response times, linear trends

335 across the task, and inertia (i.e., autoregression) in performance from trial-to-trial. In a large

336 lifespan cohort (Cam-CAN; Shafto et al., 2014), we then used this model to examine the

337 associations between these components of performance across aging and individual differences in

338 white matter microstructure. Following from a 'neural noise' hypothesis (Kail, 1997; Peters, 2009;

339 Voytek et al., 2015), we posited that reduced white matter microstructure would predict greater

340 variability in behavioral performance, reflecting a less consistent signal-to-noise ratio in the

341 propagation of speeded responses in the task (Dave et al., 2018; Kievit et al., 2016; Peters, 2009).

342 We found evidence that poorer white matter microstructure - measured through fractional

343 anisotropy - predicted increased variability, as well as decreased mean level performance (Figure

344 2) and these relationships held when controlling for age (Figure 5). Overall, these results highlight

345 the promise of approaches that directly incorporate variability as a parameter in the model, with

346 potentially unique etiological mechanisms and consequences. 
One of the primary benefits of adopting a DSEM framework here is the ability to directly

350 model within- and between-person features that are often treated as noise in simpler analyses (e.g.,

351 variability and trend), as well as complex temporal features of performance (e.g., autoregression)

352 that would be invisible to those models. Doing so not only allows us to de-confound measures of

353 mean performance and variability (de Haan-Rietdijk et al., 2016), but to model individual

354 differences in all four components of performance that can show unique patterns of relationship

355 with other individual differences measures. In each of the three tasks, model comparisons

356 overwhelmingly favored models with random effects of the four components over a model with

357 fixed effects, suggesting that human behavioral performance cannot be captured adequately by

358 models that do not incorporate individual differences. Even in reaction time data, which often has

359 a strong correlation between mean performance and variability due to floor effects on RT, DSEM

360 was able to model individual differences in these components with reasonably low correlation $(r$ $361=0.397)$.

The DSEM approach to modeling variability also allowed us to explore the relationships

363 between the random effects of the four behavioral components and observed measures of 364 interindividual variability calculated directly from the trial-level data. We showed that the iSD 365 measure was strongly related to variability $(\psi)$ from the DSEM, but with significant contributions 366 from the other random effects, while iCV was most strongly related to the mean performance factor $367(\mu)$ and negatively related to both the mean and variability factors (Figure 3 ). Taken together, these 368 results highlight the strength of the DSEM to separate out these confounded factors of performance 369 into unique factors and cautions us against using simple observed measures of variability without 370 taking steps to ensure that they are not confounded by other sources of individual differences (e.g., 371 differential trends or autoregression). 
Poorer white matter microstructure predicts slower and more variable reaction time performance

The DSEM framework presented here has the potential for many additional expansions

373 that can help to broaden our understanding of the role that variability plays in a wide range of

374 phenomenon. Two such extensions include showing that individual differences in variability have

375 unique consequences by including distal outcomes from longitudinal data into this model. Another

376 exciting extension would be to incorporate the between-person level of the DSEM into a standard

377 growth model (Hancock et al., 2001) to map trajectories of individual differences in mean

378 performance and variability. This type of approach could help to disentangle within- from

379 between-person differences in variability that we found across age in the current investigation. The

380 flexibility of DSEM to incorporate these, and other, complex effects make it a powerful tool for

381 understanding the interplay between individual differences in mean performance and variability

382 across the lifespan.

383

\section{Testing a Neural Noise Hypothesis}

385 Neural noise has been hypothesized to play a role in age-related changes in motor (Sosnoff

386 \& Newell, 2011; Voytek et al., 2015), sensory (Tran et al., 2020), and cognitive (Pertermann et

387 al., 2019; Voytek et al., 2015) abilities. Neural noise has also been proposed as a mechanism in

388 various non-normative states like ADHD (Hearne et al., 2021) and dyslexia (Frey et al., 2019).

389 Common between these different theories is the idea that noisy firing in neural networks impairs

390 consistent and efficacious behavior. Kail (1997), proposed that white matter impairment offered a

391 potential structural mechanism for increase in neural noise across aging, which we tested in the 392 current study. White matter has also been shown to be important for predicting response time

393 variability associated with attentional problems in adolescence, providing additional evidence that

394 this is a theoretically plausible target for investigation (Wiker et al., 2022). 
We utilized the dynamic structural equation model to test our hypothesis that reduced white matter microstructure would predict less consistent behavioral performance. To do so, we entered

397 each of the 10 white matter tracts (Hua et al., 2008; Kievit et al., 2016) in separate models as a 398 predictor of each of the four components of behavior. Prior work had shown consistent evidence 399 that enhanced white matter measures related to faster overall performance (Fjell et al., 2011; 400 Turken et al., 2008) and observed measures of variability (Tamnes et al., 2012). Here, we confirm 401 these prior findings, showing that white matter FA predicts both slower mean RT performance and 402 heightened variability in reaction time. These results held across the different white matter tracts, 403 with the exception of the ventral cingulate gyrus (CINGHipp), which showed a non-significant 404 effect. White matter FA did not predict individual differences in the trend or autoregressive effects, 405 although we have much less power to detect effects in the autoregressive parameter as it depends 406 on complex temporal information. While further work remains to incorporate direct measures of 407 neural oscillations into this model, this provides consistent evidence with prior 408 electrophysiological data where greater neural noise is related to poorer performance (Dave et al., 409 2018; Tran et al., 2020; Voytek et al., 2015), increases during aging (Nobukawa et al., 2019; 410 Voytek \& Knight, 2015), and is correlated with white matter morphology (Smit et al., 2012; van 411 Straaten et al., 2015).

412 To establish the specificity of these relationships, we introduced age into the model as an 413 additional predictor to control for the systematic differences in white matter FA and associated 414 changes in speeded responses across the adult lifespan (Bartzokis et al., 2010). In this full 415 conditional model, we show that the relationships between white matter and the mean and 416 variability factors are preserved, although somewhat attenuated. Consistent with prior work, 417 increased age and reduced white matter FA was associated with poorer (i.e., slower and more 
Poorer white matter microstructure predicts slower and more variable reaction time performance

418 variable) performance (Figure 5). In sum, these results provide additional support for the neural 419 noise hypothesis (Kail, 1997), reflecting a role for white matter in the signal propagation needed

420 for consistent performance and performance deficits when these fibers are compromised.

\section{Directions for Future Research}

While there are many strengths to the current approach taken here, there are several 424 avenues for further testing of the neural noise hypothesis that should be addressed in future 425 research. First, longitudinal measures of white matter tract and behavioral change over time would 426 allow us assess within-person dynamic relationships across aging. Additionally, while we tested

427 the relationship between structure and behavioral output variability, an important follow-up test of 428 the neural noise hypothesis would be to link structural brain changes during aging to evoked neural 429 responses during behavior. Combining functional recordings, either fMRI (Garrett et al., 2021) or 430 electrophysiological (McIntosh et al., 2008; Woolnough et al., 2022), with structural measures 431 (e.g., Hearne et al., 2021) would shed important light onto the consequences of white matter loss

432 for function and behavior. One strength of the DSEM approach would be to combine timeseries 433 models for both recordings and behavior and link them at the factor level to measurements of 434 structural WM, or to important cognitive outcomes across aging.

435 When studying the broader construct of variability, it is important to note that the adaptive 436 versus maladaptive nature of variability is context dependent. In the current work, variability of 437 speeded responses on a relatively simple RT appears to reflect performance deficits. However, in 438 other contexts, such as learning (McCormick \& Telzer, 2017) or more complex tasks (Garrett et 439 al., 2011; McIntosh et al., 2008), variability may allow for greater flexibility in representing the 440 task space. Understanding when variability is related to improvements versus declines in cognitive 
441 performance will offer greater insights into the mechanistic processes underlying lifespan brain 442 development.

\section{Conclusions}

445 The study of variability as an important marker of individual differences in behavior and 446 cognition is still in the early phases of development. We presented here a powerful framework for 447 modeling variability in concert with other components of behavior, including mean performance, 448 trends over time, and inertia in trial-level performance. We further show that these individual 449 differences can be used to test a theory of neural noise by including person-level predictors and 450 found that both reduced white matter microstructure and increased age uniquely predict poorer 451 task performance, consistent with the importance of intact white matter tracts for appropriate signal 452 propagation. The results here highlight the promise of leveraging advanced behavioral modeling 453 to move beyond a sole focus on mean differences and recognize variability as an important 454 individual difference marker for understanding human behavior. By combining these models with 455 measures of brain structure and function, this framework can be used to test a wide array of 456 hypotheses for how these individual differences variability emerge. 
Poorer white matter microstructure predicts slower and more variable reaction time performance

Acknowledgements

459 RAK is supported by a Hypatia Fellowship at the RadboudUMC.

461 The Cambridge Centre for Ageing and Neuroscience (Cam-CAN) research was supported by the 462 Biotechnology and Biological Sciences Research Council (BB/H008217/1). The Cam-CAN 463 corporate author consists of the Project principal personnel: Lorraine K Tyler, Carol Brayne, 464 Edward T Bullmore, Andrew C Calder, Rhodri Cusack, Tim Dalgleish, John Duncan, Richard N 465 Henson, Fiona E Matthews, William D Marslen-Wilson, James B Rowe, Meredith A Shafto; 466 Research Associates: Karen Campbell, Teresa Cheung, Simon Davis, Linda Geerligs, Rogier 467 Kievit, Anna McCarrey, Abdur Mustafa, Darren Price, David Samu, Jason R Taylor, Matthias 468 Treder, Kamen A Tsvetanov, Janna van Belle, Nitin Williams; Research Assistants: Lauren 469 Bates, Tina Emery, Sharon Erzinçlioglu, Andrew Gadie, Sofia Gerbase, Stanimira Georgieva, 470 Claire Hanley, Beth Parkin, David Troy; Affiliated Personnel: Tibor Auer, Marta Correia, Lu 471 Gao, Emma Green, Rafael Henriques; Research Interviewers: Jodie Allen, Gillian Amery, Liana 472 Amunts, Anne Barcroft, Amanda Castle, Cheryl Dias, Jonathan Dowrick, Melissa Fair, Hayley 473 Fisher, Anna Goulding, Adarsh Grewal, Geoff Hale, Andrew Hilton, Frances Johnson, Patricia 474 Johnston, Thea Kavanagh-Williamson, Magdalena Kwasniewska, Alison McMinn, Kim

475 Norman, Jessica Penrose, Fiona Roby, Diane Rowland, John Sargeant, Maggie Squire, Beth 476 Stevens, Aldabra Stoddart, Cheryl Stone, Tracy Thompson, Ozlem Yazlik; and administrative 477 staff: Dan Barnes, Marie Dixon, Jaya Hillman, Joanne Mitchell, Laura Villis, Ethan Knights. 


\section{References}

Adam, K. C. S., Mance, I., Fukuda, K., \& Vogel, E. K. (2015). The Contribution of Attentional Lapses to Individual Differences in Visual Working Memory Capacity. Journal of Cognitive Neuroscience, 27(8), 1601-1616. https://doi.org/10.1162/jocn_a_00811

Asparouhov, T., Hamaker, E. L., \& Muthén, B. (2018). Dynamic Structural Equation Models. Structural Equation Modeling: A Multidisciplinary Journal, 25(3), 359-388. https://doi.org/10.1080/10705511.2017.1406803

Bartzokis, G., Lu, P. H., Tingus, K., Mendez, M. F., Richard, A., Peters, D. G., Oluwadara, B., Barrall, K. A., Finn, J. P., Villablanca, P., Thompson, P. M., \& Mintz, J. (2010). Lifespan trajectory of myelin integrity and maximum motor speed. Neurobiology of Aging, 31(9), 1554-1562. https://doi.org/10.1016/j.neurobiolaging.2008.08.015

Bennett, I. J., \& Madden, D. J. (2014). Disconnected aging: Cerebral white matter integrity and age-related differences in cognition. Neuroscience, 276, 187-205. https://doi.org/10.1016/j.neuroscience.2013.11.026

Britton, T. C., Meyer, B.-U., \& Benecke, R. (1991). Variability of cortically evoked motor responses in multiple sclerosis. Electroencephalography and Clinical Neurophysiology/Evoked Potentials Section, 81(3), 186-194. https://doi.org/10.1016/0168-5597(91)90071-5

497 Bunce, D., Anstey, K., Christensen, H., Dear, K., Wen, W., \& Sachdev, P. (2007). White matter hyperintensities and within-person variability in community-dwelling adults aged 60-64 
Poorer white matter microstructure predicts slower and more variable reaction time performance

years. Neuropsychologia, 45(9), 2009-2015.

https://doi.org/10.1016/j.neuropsychologia.2007.02.006

Dave, S., Brothers, T. A., \& Swaab, T. Y. (2018). 1/f neural noise and electrophysiological indices of contextual prediction in aging. Brain Research, 1691, 34-43. https://doi.org/10.1016/j.brainres.2018.04.007

de Haan-Rietdijk, S., Kuppens, P., \& Hamaker, E. L. (2016). What's in a Day? A Guide to Decomposing the Variance in Intensive Longitudinal Data. Frontiers in Psychology, 7, 891. https://doi.org/10.3389/fpsyg.2016.00891

de Mooij, S. M. M., Henson, R. N. A., Waldorp, L. J., \& Kievit, R. A. (2018). Age Differentiation within Gray Matter, White Matter, and between Memory and White Matter in an Adult Life Span Cohort. The Journal of Neuroscience: The Official Journal of the Society for Neuroscience, 38(25), 5826-5836. https://doi.org/10.1523/JNEUROSCI.1627-17.2018

Dykiert, D., Der, G., Starr, J. M., \& Deary, I. J. (2012). Age Differences in Intra-Individual Variability in Simple and Choice Reaction Time: Systematic Review and Meta-Analysis. PLOS ONE, 7(10), e45759. https://doi.org/10.1371/journal.pone.0045759

Eizenman, D. R., Nesselroade, J. R., Featherman, D. L., \& Rowe, J. W. (1997). Intraindividual variability in perceived control in a older sample: The MacArthur successful aging studies. Psychology and Aging, 12(3), 489-502. https://doi.org/10.1037/08827974.12.3.489

Fiske, D. W., \& Rice, L. (1955). Intra-individual response variability. Psychological Bulletin, 52(3), 217-250. https://doi.org/10.1037/h0045276 
520 Fjell, A. M., Westlye, L. T., Amlien, I. K., \& Walhovd, K. B. (2011). Reduced White Matter Integrity Is Related to Cognitive Instability. Journal of Neuroscience, 31(49), 1806018072. https://doi.org/10.1523/JNEUROSCI.4735-11.2011

523

Folstein, M. F., Folstein, S. E., \& McHugh, P. R. (1975). “Mini-mental state”. Journal of Psychiatric Research, 12(3), 189-198. https://doi.org/10.1016/0022-3956(75)90026-6

Frey, A., François, C., Chobert, J., Besson, M., \& Ziegler, J. C. (2019). Behavioral and electrophysiological investigation of speech perception deficits in silence, noise and envelope conditions in developmental dyslexia. Neuropsychologia, 130, 3-12. https://doi.org/10.1016/j.neuropsychologia.2018.07.033

Gamaldo, A. A., An, Y., Allaire, J. C., Kitner-Triolo, M. H., \& Zonderman, A. B. (2012). Variability in performance: Identifying early signs of future cognitive impairment. Neuropsychology, 26(4), 534-540. https://doi.org/10.1037/a0028686

Garrett, D. D., Kovacevic, N., McIntosh, A. R., \& Grady, C. L. (2011). The Importance of Being Variable. Journal of Neuroscience, 31(12), 4496-4503. https://doi.org/10.1523/JNEUROSCI.5641-10.2011

Garrett, D. D., Skowron, A., Wiegert, S., Adolf, J., Dahle, C. L., Lindenberger, U., \& Raz, N. (2021). Lost Dynamics and the Dynamics of Loss: Longitudinal Compression of Brain Signal Variability is Coupled with Declines in Functional Integration and Cognitive Performance. Cerebral Cortex, 31(11), 5239-5252. https://doi.org/10.1093/cercor/bhab154

Gelman, A., \& Rubin, D. B. (1992). Inference from Iterative Simulation Using Multiple Sequences. Statistical Science, 7(4). https://doi.org/10.1214/ss/1177011136 
Poorer white matter microstructure predicts slower and more variable reaction time performance

541 Halliday, D. W. R., Gawryluk, J. R., Garcia-Barrera, M. A., \& MacDonald, S. W. S. (2019). White Matter Integrity Is Associated With Intraindividual Variability in Neuropsychological Test Performance in Healthy Older Adults. Frontiers in Human Neuroscience, 13, 352. https://doi.org/10.3389/fnhum.2019.00352

Hancock, G. R., Kuo, W.-L., \& Lawrence, F. R. (2001). An Illustration of Second-Order Latent Growth Models. Structural Equation Modeling: A Multidisciplinary Journal, 8(3), 470489.

Haynes, B. I., Bauermeister, S., \& Bunce, D. (2017). A Systematic Review of Longitudinal Associations Between Reaction Time Intraindividual Variability and Age-Related Cognitive Decline or Impairment, Dementia, and Mortality. Journal of the International

Hearne, L. J., Lin, H.-Y., Sanz-Leon, P., Tseng, W.-Y. I., Gau, S. S.-F., Roberts, J. A., \& Cocchi, L. Neuropsychological Society, 23(5), 431-445. https://doi.org/10.1017/S1355617717000236

Hua, K., Zhang, J., Wakana, S., Jiang, H., Li, X., Reich, D. S., Calabresi, P. A., Pekar, J. J., van Zijl, P. (2021). ADHD symptoms map onto noise-driven structure-function decoupling between hub and peripheral brain regions. Molecular Psychiatry, 26(8), 4036-4045. C. M., \& Mori, S. (2008). Tract probability maps in stereotaxic spaces: Analyses of white matter anatomy and tract-specific quantification. Neurolmage, 39(1), 336-347. https://doi.org/10.1016/j.neuroimage.2007.07.053 
561 Jones, D. K., Knösche, T. R., \& Turner, R. (2013). White matter integrity, fiber count, and other

562

563

564

565

566

567

568

569

570

571

572

573

574

575

576

577

578

579

580

581

fallacies: The do's and don'ts of diffusion MRI. Neurolmage, 73, 239-254.

https://doi.org/10.1016/j.neuroimage.2012.06.081

Kail, R. (1997). The neural noise hypothesis: Evidence from processing speed in adults with multiple sclerosis. Aging, Neuropsychology, and Cognition, 4(3), 157-165. https://doi.org/10.1080/13825589708256644

Kievit, R. A., Davis, S. W., Griffiths, J., Correia, M. M., Cam-CAN, \& Henson, R. N. (2016). A watershed model of individual differences in fluid intelligence. Neuropsychologia, 91, 186-198. https://doi.org/10.1016/j.neuropsychologia.2016.08.008

Lavery, M. R., Acharya, P., Sivo, S. A., \& Xu, L. (2019). Number of predictors and multicollinearity: What are their effects on error and bias in regression? Communications in Statistics - Simulation and Computation, 48(1), 27-38. https://doi.org/10.1080/03610918.2017.1371750

Li, S.-C., \& Lindenberger, U. (1999). Cross-level unification: A computational exploration of the link between deterioration of neurotransmitter systems and dedifferentiation of cognitive abilities in old age. In Cognitive Neuroscience of Memory (pp. 103-146). Hogrefe \& Huber Publishers.

Li, S.-C., Lindenberger, U., Hommel, B., Aschersleben, G., Prinz, W., \& Baltes, P. B. (2004). Transformations in the Couplings Among Intellectual Abilities and Constituent Cognitive Processes Across the Life Span. Psychological Science, 15(3), 155-163. https://doi.org/10.1111/j.0956-7976.2004.01503003.x 
Poorer white matter microstructure predicts slower and more variable reaction time performance

582

583

584

585

586

587

588

589

590

591

592

593

594

595

596

597

598

599

600

601

602

MacDonald, S. W. S., Nyberg, L., \& Bäckman, L. (2006). Intra-individual variability in behavior: Links to brain structure, neurotransmission and neuronal activity. Trends in Neurosciences, 29(8), 474-480. https://doi.org/10.1016/j.tins.2006.06.011

McCormick, E. M. (2021). Multi-Level Multi-Growth Models: New opportunities for addressing developmental theory using advanced longitudinal designs with planned missingness. Developmental Cognitive Neuroscience, 51, 101001. https://doi.org/10.1016/j.dcn.2021.101001

McCormick, E. M., \& Telzer, E. H. (2017). Adaptive Adolescent Flexibility: Neurodevelopment of Decision-making and Learning in a Risky Context. J. Cogn. Neurosci., 29(3), 413-423.

McIntosh, A. R., Kovacevic, N., \& Itier, R. J. (2008). Increased Brain Signal Variability Accompanies Lower Behavioral Variability in Development. PLOS Computational Biology, 4(7), e1000106. https://doi.org/10.1371/journal.pcbi.1000106

McNeish, D., \& Hamaker, E. L. (2020). A primer on two-level dynamic structural equation models for intensive longitudinal data in Mplus. Psychological Methods, 25(5), 610-635. https://doi.org/10.1037/met0000250

Nesselroade, J. (1991). The Warp and Woof of the Developmental Fabric.

Nobukawa, S., Kikuchi, M., \& Takahashi, T. (2019). Changes in functional connectivity dynamics with aging: A dynamical phase synchronization approach. Neurolmage, 188, 357-368. https://doi.org/10.1016/j.neuroimage.2018.12.008

Pertermann, M., Mückschel, M., Adelhöfer, N., Ziemssen, T., \& Beste, C. (2019). On the interrelation of $1 / f$ neural noise and norepinephrine system activity during motor 
response inhibition. Journal of Neurophysiology, 121(5), 1633-1643. https://doi.org/10.1152/jn.00701.2018

Peters, A. (2009). The effects of normal aging on myelinated nerve fibers in monkey central nervous system. Frontiers in Neuroanatomy. http://dx.doi.org/10.3389/neuro.05.011.2009

Salthouse, T. A. (1996). The processing-speed theory of adult age differences in cognition. Psychological Review, 103(3), 403-428. https://doi.org/10.1037/0033-295X.103.3.403

Schuurman, N. K., Ferrer, E., de Boer-Sonnenschein, M., \& Hamaker, E. L. (2016). How to compare cross-lagged associations in a multilevel autoregressive model. Psychological

Shafto, M. A., Tyler, L. K., Dixon, M., Taylor, J. R., Rowe, J. B., Cusack, R., Calder, A. J., MarslenWilson, W. D., Duncan, J., Dalgleish, T., Henson, R. N., Brayne, C., \& Matthews, F. E. (2014). The Cambridge Centre for Ageing and Neuroscience (Cam-CAN) study protocol: A cross-sectional, lifespan, multidisciplinary examination of healthy cognitive ageing.

Smit, D. J. A., Boersma, M., Schnack, H. G., Micheloyannis, S., Boomsma, D. I., Pol, H. E. H., Stam, C. J., \& Geus, E. J. C. de. (2012). The Brain Matures with Stronger Functional https://doi.org/10.1371/journal.pone.0036896

Sorg, S. F., Merritt, V. C., Clark, A. L., Werhane, M. L., Holiday, K. A., Schiehser, D. M., Bondi, M., \& Delano-Wood, L. (2021). Elevated Intraindividual Variability in Executive Functions and Associations with White Matter Microstructure in Veterans with Mild Traumatic 
Poorer white matter microstructure predicts slower and more variable reaction time performance

625

626

627

628

629

630

631

632

633

634

635

636

638

639

640

641

642

643

644

645

Brain Injury. Journal of the International Neuropsychological Society, 27(4), 305-314. https://doi.org/10.1017/S1355617720000879

Sosnoff, J. J., \& Newell, K. M. (2011). Aging and motor variability: A test of the neural noise hypothesis. Experimental Aging Research, 37(4), 377-397.

https://doi.org/10.1080/0361073X.2011.590754

Tamnes, C. K., Fjell, A. M., Westlye, L. T., Ostby, Y., \& Walhovd, K. B. (2012). Becoming Consistent: Developmental Reductions in Intraindividual Variability in Reaction Time Are Related to White Matter Integrity. Journal of Neuroscience, 32(3), 972-982. https://doi.org/10.1523/JNEUROSCI.4779-11.2012

Taylor, J. R., Williams, N., Cusack, R., Auer, T., Shafto, M. A., Dixon, M., Tyler, L. K., Cam-CAN, \& Henson, R. N. (2017). The Cambridge Centre for Ageing and Neuroscience (Cam-CAN) data repository: Structural and functional MRI, MEG, and cognitive data from a crosssectional adult lifespan sample. Neurolmage, 144, 262-269. https://doi.org/10.1016/j.neuroimage.2015.09.018

Tran, T. T., Rolle, C. E., Gazzaley, A., \& Voytek, B. (2020). Linked sources of neural noise contribute to age-related cognitive decline. Journal of Cognitive Neuroscience, 32(9), 1813-1822. https://doi.org/10.1162/jocn_a_01584

Turken, U., Whitfield-Gabrieli, S., Bammer, R., Baldo, J. V., Dronkers, N. F., \& Gabrieli, J. D. E. (2008). Cognitive processing speed and the structure of white matter pathways: Convergent evidence from normal variation and lesion studies. Neurolmage, 42(2), 1032-1044. https://doi.org/10.1016/j.neuroimage.2008.03.057 
646

647

648

van Straaten, E. C. W., den Haan, J., de Waal, H., van der Flier, W. M., Barkhof, F., Prins, N. D., \& Stam, C. J. (2015). Disturbed phase relations in white matter hyperintensity based vascular dementia: An EEG directed connectivity study. Clinical Neurophysiology: Official Journal of the International Federation of Clinical Neurophysiology, 126(3), 497-504. https://doi.org/10.1016/j.clinph.2014.05.018

Voytek, B., \& Knight, R. T. (2015). Dynamic Network Communication as a Unifying Neural Basis for Cognition, Development, Aging, and Disease. Biological Psychiatry, 77(12), 10891097. https://doi.org/10.1016/j.biopsych.2015.04.016

Voytek, B., Kramer, M. A., Case, J., Lepage, K. Q., Tempesta, Z. R., Knight, R. T., \& Gazzaley, A. (2015). Age-Related Changes in 1/f Neural Electrophysiological Noise. Journal of Neuroscience, 35(38), 13257-13265. https://doi.org/10.1523/JNEUROSCI.2332-14.2015

Wang, L. (Peggy), \& Grimm, K. J. (2012). Investigating Reliabilities of Intraindividual Variability Indicators. Multivariate Behavioral Research, 47(5), 771-802. https://doi.org/10.1080/00273171.2012.715842

Williams, B. R., Hultsch, D. F., Strauss, E. H., Hunter, M. A., \& Tannock, R. (2005). Inconsistency in Reaction Time Across the Life Span. Neuropsychology, 19(1), 88-96. https://doi.org/10.1037/0894-4105.19.1.88

Woodrow, H. (1932). Quotidian variability. Psychological Review, 39(3), 245-256. https://doi.org/10.1037/h0073076

Woolnough, O., Kadipasaoglu, C. M., Conner, C. R., Forseth, K. J., Rollo, P. S., Rollo, M. J., Baboyan, V. G., \& Tandon, N. (2022). Dataset of human intracranial recordings during 
Poorer white matter microstructure predicts slower and more variable reaction time performance

667 famous landmark identification. Scientific Data, 9(1), Article 1.

668 https://doi.org/10.1038/s41597-022-01125-8

669 